\title{
Novel Rat Model for Neurocysticercosis Using Taenia solium
}

Manuela R. Verastegui, ${ }^{* \dagger}$ Alan Mejia, ${ }^{* \dagger}$ Taryn Clark, ${ }^{\star \ddagger}$ Cesar M. Gavidia, ${ }^{\star \S}$ Javier Mamani, ${ }^{*}$ Fredy Ccopa, ${ }^{* \dagger}$ Noelia Angulo, ${ }^{* \dagger}$ Nancy Chile, ${ }^{* \dagger}$ Rogger Carmen, ${ }^{* \dagger}$ Roxana Medina, ${ }^{* \|}$ Hector H. García, ${ }^{* * *}$ Silvia Rodriguez, ${ }^{* * *}$ Ynes Ortega, ${ }^{* \dagger \dagger}$ and Robert H. Gilman* $* \sharp \S \S$

\begin{abstract}
From the Cysticercosis Working Group in Peru*; Infectious Diseases Laboratory Research-LID, ${ }^{\dagger}$ Faculty of Science and Philosophy, Alberto Cazorla Talleri, and the Faculty of Veterinary Medicine and Animal Husbandry, ${ }^{\natural}$ Universidad Peruana Cayetano Heredia, Lima, Peru; the Weill Cornell Medical College, ${ }^{\ddagger}$ New York, New York; the Public Health Section, ${ }^{\S}$ School of Veterinary Medicine, Universidad Nacional Mayor de San Marcos, Lima, Peru; the Department of Biology Science," Universidad Nacional del Altiplano, Puno, Peru; the Cysticercosis Unit, ** Instituto de Ciencias Neurologicas, Lima, Peru; the Department of Food Science \& Technology, ${ }^{\dagger \dagger}$ The University of Georgia, Athens, Georgia; the Department of International Health, ${ }^{\ddagger \ddagger}$ Bloomberg School of Hygiene and Public Health, Johns Hopkins University, Baltimore, Maryland; and the Asociación Benéfica PRISMA ${ }^{\S \S}$ San Miguel, Lima, Peru
\end{abstract}

\author{
Accepted for publication \\ April 23, 2015. \\ Address correspondence to \\ Manuela R. Verastegui, Ph.D., \\ Infectious Diseases Laboratory \\ Research-LID, Department of \\ Cellular and Molecular \\ Biology, Faculty of Science and \\ Philosophy Alberto Cazorla \\ Talleri, Universidad Peruana \\ Cayetano Heredia, Av. Honorio \\ Delgado 430, Urbanizacion \\ Ingenieria, San Martin de \\ Porres, Lima 31, Peru. E-mail: \\ manuela.verastegui@upch.pe.
}

\begin{abstract}
Neurocysticercosis is caused by Taenia solium infecting the central nervous system and is the leading cause of acquired epilepsy and convulsive conditions worldwide. Research into the pathophysiology of the disease and appropriate treatment is hindered by lack of cost-effective and physiologically similar animal models. We generated a novel rat neurocysticercosis model using intracranial infection with activated $T$. solium oncospheres. Holtzman rats were infected in two separate groups: the first group was inoculated extraparenchymally and the second intraparenchymally, with different doses of activated oncospheres. The groups were evaluated at three different ages. Histologic examination of the tissue surrounding $T$. solium cysticerci was performed. Results indicate that generally infected rats developed cysticerci in the brain tissue after 4 months, and the cysticerci were observed in the parenchymal, ventricle, or submeningeal brain tissue. The route of infection did not have a statistically significant effect on the proportion of rats that developed cysticerci, and there was no dependence on infection dose. However, rat age was crucial to the success of the infection. Epilepsy was observed in $9 \%$ of rats with neurocysticercosis. In histologic examination, a layer of collagen tissue, inflammatory infiltrate cells, perivascular infiltrate, angiogenesis, spongy change, and mass effect were observed in the tissue surrounding the cysts. This study presents a suitable animal model for the study of human neurocysticercosis. (Am J Pathol 2015, 185: 2259-2268; http://dx.doi.org/ 10.1016/j.ajpath.2015.04.015)
\end{abstract}

Neurocysticercosis (NCC) is caused by the tapeworm Taenia solium when it infects the central nervous system $(\mathrm{CNS})^{1}$ and is the leading cause of acquired epilepsy and convulsive conditions worldwide. ${ }^{1-4}$ The life cycle of $T$. solium involves the pig as the common intermediate host harboring the larval vesicles (metacestode or cysticerci); humans enter the life cycle as an unintentional intermediate host. Taeniasis develops after ingestion of pork containing live cysticerci, which develop into adult tapeworms in the small intestine. Eggs produced by the tapeworm are released in the feces of the human host. $T$. solium eggs contain the embryonic form of the parasite, known as the oncosphere. Ingestion of fecal contaminated food or water allows the larvae to migrate to the brain, where they transform into cysticerci and can live within the CNS for several years. ${ }^{5}$
NCC is the most prevalent neurologic disease in developing countries where pigs are raised because there are often poor sanitary conditions that allow entry of $T$. solium eggs into the food chain. $^{6-9}$

Although there have been numerous studies of the pathology and clinical evolution of NCC, the pathogenesis of the infection remains poorly elucidated. The onset of the symptoms starts

\footnotetext{
Supported by Bill and Melinda Gates Foundation Cysticercosis Elimination in Peru grants 23981 and 33848 (H.H.G.), NIH grant 5D43TW006581 (Infectious Diseases Training Program in Peru) (R.H.G.), NIH training grant FIC/NIH D43 TW001140, and Fondo para la Innovación, Ciencia y Tecnología FINCyT grant 129-FINCyT-IB-2013 (M.R.V.). Disclosures: None declared.
} 
many years after exposure and initial infection, most likely when the cysts begin to degenerate and an inflammatory response is activated in the host. ${ }^{10,11}$ The mechanisms of initial infection, cyst development, activation of the immune response, and the resulting inflammatory pathways are difficult to study in humans because of the CNS location of the cysts and the slow progression of the disease.

A cheap and accurate animal model of NCC using $T$. solium as the primary infecting agent would provide an effective means for studying this disease. A pig model has been previously described, ${ }^{12-14}$ and although pigs are the natural intermediate hosts, the porcine model has significant disadvantages. Experimental infection of the pig CNS with $T$. solium is difficult because of the uncertainty and variability of the oral infection efficacy, the cost of purchasing and maintaining the pigs for a long time, and the paucity of commercial reagents available for the detection of inflammatory biomarkers in pigs.

Other NCC animal models were developed using mice that had been infected intracranially with Taenia crassiceps $^{15,16}$ or Mesocestoides corti. ${ }^{17,18}$ In both models, researchers used the metacestode stage to infect the mice, whereas in NCC, the natural infective stage of the T. solium life cycle is the oncosphere stage. Both metacestode infections have serious limitations; it is not possible in either model to test different schemes of diagnosis or therapies relevant to human NCC. Therefore, in the present study, we tested the hypothesis that the rat can be a useful animal model for NCC using intracranial infection with activated T. solium oncospheres.

\section{Material and Methods}

\section{Animals}

Outbred Holtzman rats 10 to 26 days old were purchased from the animal facility of the Universidad Peruana Cayetano Heredia (Lima, Peru). Animal experiments were conducted according to the ethical guidelines of the Universidad Peruana Cayetano Heredia System. Rats had access to food and water ad libitum and were kept on a 12-hour light/dark cycle.

\section{T. solium Oncosphere Preparation}

Adult T. solium tapeworms were collected from human patients following the niclosamide regimen. ${ }^{19}$ Species differentiation was determined by histologic analysis (parasite morphologic analysis) and PCR with restriction fragment length polymorphism, as previously reported. ${ }^{20}$ Eggs were obtained from gravid proglottids and incubated in sodium hypochlorite $(0.75 \%)$ for 10 minutes at $4^{\circ} \mathrm{C}$ to facilitate in vitro hatching of oncospheres. These oncospheres were activated using artificial intestinal fluid (pancreatine 1\%, fresh porcine bile $1 \%, \mathrm{NaHCO}_{3} 0.2 \%$ in RPMI 1640 medium, $\mathrm{pH} 8.04$ ) at $37^{\circ} \mathrm{C}$ for 1 hour. $^{21}$

\section{Experimental Rat Intracranial Infections}

To standardize and evaluate the rat model of NCC, we performed three experiments: infection dose and brain anatomical point of inoculation, rat age at infection, and parasite viability by in vitro cyst evagination assessment. We then further analyzed the CNS tissue and cysts and performed cysticercosis antibody detection, cysticercosis antigen detection, and histopathologic studies.

Infection Dose and Brain Anatomical Point of Inoculation Our aim was to test the brain anatomical point of inoculation and the $T$. solium-activated oncosphere infection dose. Two types of anatomical site of inoculation infection were evaluated. Type 1 was the extraparenchymal cerebral inoculation, where the needle was inserted to a depth of $2 \mathrm{~mm}$ at the junction of the superior sagittal and the transverse sutures (bregma). This approach allowed insertion of the needle into a protective cuff, avoiding penetration of brain tissue. Type -2 is the intraparenchymal cerebral inoculation, where the needle was inserted to a depth of $4 \mathrm{~mm}$, using the same technique described above. In this case, the needle was inserted with sufficient depth to penetrate brain parenchymal tissue. Control rats for the two types of inoculation were injected with $100 \mu \mathrm{L}$ of sterile physiological saline solution. Before intracranial inoculation, the rats were anesthetized with $70 \mathrm{mg} / \mathrm{kg}$ of ketamine plus $11 \mathrm{mg} / \mathrm{kg}$ of xylazine.

Holtzman rats aged 16 to 18 days were infected intracranially with different numbers of activated $T$. solium oncospheres suspended in $100 \mu \mathrm{L}$ of $0.877 \%$ sterile physiologic salt solution using a $1-\mathrm{mL}$ syringe with a 25 -gauge needle. ${ }^{17}$ In the initial study, we aimed to find a reference dose. Four groups of three rats each were infected with 10, 20,30, and 40 activated oncospheres following the extraparenchymal inoculation procedure (type 1) as described above.

Another study, based on the results of the first one, evaluated different anatomical locations of infection and infection dose to determine which led to the highest proportion of infected rat brain tissue. Six groups of six rats each were inoculated extraparenchymally (type 1) with 30, $60,90,180,360$, or 720 activated oncospheres, respectively; another six rats were used as controls and were injected with sterile physiological salt solution $(0.877 \%)$. Another batch of 42 rats (six groups injected with oncospheres and one control group) was also inoculated intraparenchymally (type 2) as described above.

All animals were euthanized between 4 and 5 months after intracranial inoculation, and the extent of infection was evaluated. Animals were euthanized after being anesthetized with a mixture of $100 \mathrm{mg} / \mathrm{kg}$ of ketamine, $10 \mathrm{mg} / \mathrm{kg}$ of xylazine, and $3 \mathrm{mg} / \mathrm{kg}$ of tramadol. Blood and cerebrospinal fluid (CSF) were collected from the rats. The CSF was collected from the cistern magna using a 25 -gauge needle. ${ }^{22}$ Subsequently, the animals were perfused via the left ventricle with $300 \mathrm{~mL}$ of cold phosphate-buffered saline (PBS) and then with $200 \mathrm{~mL}$ of PBS plus 4\% paraformaldehyde. The 
brain was immediately removed, placed in PBS plus $4 \%$ paraformaldehyde for 24 hours, washed with PBS, and stored in alcohol until further use. A study of the skeletal muscle of the rats to identify cysticerci was conducted using macroscopic examination in $50 \%$ of animals.

\section{Rat Age at Infection}

To assess the significance of the age of the rats at the time of infection, three groups of rats of different ages were evaluated. A first group of 18 rats was infected at 10 days of age, a second group of nine rats at 18 days of age, and a third group of eight rats at 26 days of age. The animals were infected with 500 activated oncospheres via extraparenchymal cerebral inoculation (type 1) under the same procedures described in the previous experiment. The animals were euthanized at 5 to 6 months after infection as described above. We used three control rats for each age group, which were injected with 100 $\mu \mathrm{L}$ of sterile physiologic saline solution.

\section{Parasite Viability by in Vitro Cyst Evagination}

In vitro evagination was used to evaluate the proportion of brain cysts that were viable after experimental infection. A total of 15 rats were inoculated extraparenchymally (type 1) with 500 activated oncospheres following the technique described above; the euthanasia was performed approximately 4 months after infection. The brain was immediately removed and dissected to obtain the cysts and determine their anatomical location. In vitro cyst evagination with whole bovine bile was performed as described previously, ${ }^{23,24}$ and the presence of a moving scolex outside the cyst wall (evagination) was used to define the cyst viability.

\section{Cysticercosis Antibody Detection}

Western blot (WB) was used to detect cysticercosis antibodies in the rat's plasma samples, which were obtained before euthanasia. In brief, this assay uses a semipurified fraction that contains seven $T$. solium glycoprotein antigens (diagnostic bands GP50, GP42-39, GP24, GP21, GP18, GP14, and GP13) in an immunoblot format to detect infection-specific antibodies. Reactions to at least one band are considered positive. ${ }^{25}$

\section{Cysticercosis Antigen Detection}

Circulating parasite antigen was detected by enzyme-linked immunosorbent assay (ELISA) using the technique described by Dorney and colleagues. ${ }^{26}$ Briefly, monoclonal antibody B158C11A10 was used to capture the cyst antigen from all rat plasma samples. A sample was considered positive if the optical density was greater than a cut-off defined by analyzing the values of eight known negative samples by the $t$-test. ${ }^{26}$ In addition, we tested the CSF from three necropsy-positive rats that were inoculated with 720 activated oncospheres extraparenchymally and two controls rats (type 1).

\section{Histopathologic Study}

\section{Processing Brain Tissues}

The brain tissue was embedded in paraffin and cut into 3- $\mu \mathrm{m}-$ thick coronal sections. Serial brain sections were deparaffinized and rehydrated for standard staining with hematoxylin and eosin to see the cyst characteristics and perform histopathologic analysis. The Luna staining was used to differentiate eosinophil cells. ${ }^{12}$ Description of the location of the cysts was in agreement with Paxinos and Watson. ${ }^{27}$ Brain tissue slides that had the scolex present were evaluated; we also read all the slides from intraventricular cysts that had absent scolices.

\section{Inflammatory Infiltrated Cells}

A semiquantitative analysis of the cellular infiltrates was performed by counting the number of each cell (heterophils, lymphocytes, plasmocytes, and macrophages) in the tissue surrounding the cyst, using a microscope at $\times 40$ magnification. The area under the microscope was calculated to be $6.25 \times 10^{-2} \mathrm{~mm}^{2}$. The means \pm SEM were calculated from 12 randomly selected fields to quantify the inflammatory cells. Other pathologic alterations, such as mass effect, pyknotic cells, and meningitis, were also documented.

\section{Spongy Changes}

Spongy changes were characterized as variably sized fluidfilled spaces in the brain tissue surrounding a cyst. The severity of the brain damage surrounding the cyst was classified as follows: absent (no spongy changes observed), mild $(<20 \%$ of the brain tissue was affected), moderate ( $21 \%$ to $50 \%$ of the brain tissue affected), and severe ( $>50 \%$ of the brain tissue had alterations).

\section{Perivascular Infiltrate}

Perivascular infiltration was observed in the brain tissue surrounding the cysts. It was classified according to the proportion of perivascular infiltrate as follows: absent (no infiltration), mild $(<20 \%$ of perivascular infiltration), moderate ( $21 \%$ to $50 \%$ of perivascular infiltration), and severe ( $>50 \%$ infiltration).

\section{Collagen Layer}

Masson's trichromic stain was used to evaluate the thickness of the collagen layer surrounding the parasites at $\times 40$ magnification. The thickness, in micrometers, was measured at four selected points around the parasite in the microphotographs taken using Progress Mac Capture Pro software version 2.7.6 (Jenoptik AG, Villingen-Schwenningen, Germany). The data were recorded as means \pm SEM of the width of the collagen.

\section{Angiogenesis}

Angiogenesis was measured by means \pm SEM of the number of new small blood vessels, lined by amorphous endothelial cells, found in the fibrotic tissue and the interface between fibrotic tissue and brain parenchymal tissue surrounding the cyst. 


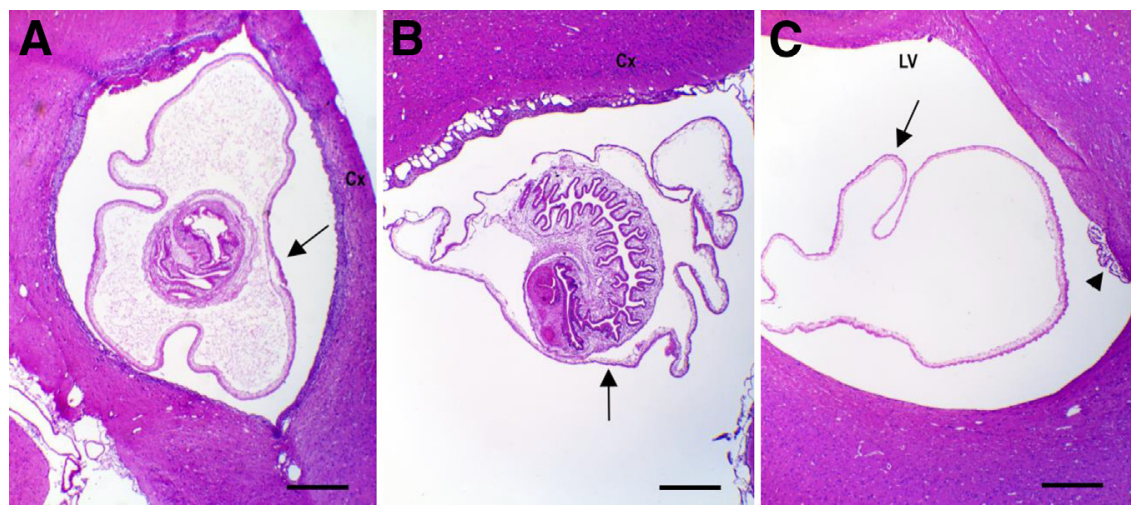

Figure 1 Taenia solium cysticercus is observed by hematoxylin and eosin in parenchyma, meninges, and ventricle of rat brain. A: Cysticercus (arrow) located in the parenchymal tissue in proximity to the cortex $(\mathrm{Cx})$. B: Cysticercus (arrow) located in submeningeal space comprising the brain cortex $(C x)$. C: Cysticercus located in the lateral ventricle (LV). Arrow indicates the teguments of cysticercus without scolex, and arrowhead indicates choroid plexus. Scale bar $=1 \mathrm{~mm}$. Original magnification: $\times 2.5$.

\section{Immunohistochemical Analysis}

After performing the rat necropsies as described above (rat age at infection), we selected four rats from the 10- and 18day-old infection group that had only one parenchymal cyst along with three control rats. The brains were immediately removed, placed in $30 \%$ sucrose for 48 hours, and then stored at $-20^{\circ} \mathrm{C}$. For each brain, three $10-\mu \mathrm{m}$ coronal sections were cut on a cryostat (Leica Microsystems, Wetzlar, Germany) at $-20^{\circ} \mathrm{C}$ and mounted on slides for immunohistochemical analysis. ${ }^{28}$ Vascular endothelial growth factor (VEGF) expression was measured to evaluate angiogenesis. ${ }^{29,30}$ The brain sections were washed with PBS, blocked with 5\% goat normal sera in PBS 0.05\% Tween 20 (PBS-T), and incubated overnight at $4{ }^{\circ} \mathrm{C}$ with $1: 100$ rabbit antiVEGF (Abcam, Cambridge, MA) and 5\% goat normal sera in PBS-T. The sections were washed with PBS-T three times and incubated with 1:250 conjugated Alexa fluor 594 affinity-purified goat anti-rabbit IgG (Life Technologies, Woburn, MA). Stained sections were coverslipped using ProLong Gold Antifade Mountant with DAPI (Life Technologies) and observed at $590 \mathrm{~nm}$ with an inverted microscope (Axio Observed A1; Zeiss, Oberkochen, Germany). Images were obtained with an Axiocam MRC camera (Zeiss) with Zen Pro Software version 2012. Adobe Photoshop CC 2014 was used for final image processing.

In addition, we evaluated the blood brain barrier (BBB) disruption by rat $\operatorname{IgG}$ extravasation observed in the brain parenchyma. ${ }^{28,29}$ Tissue sections of $3-\mu \mathrm{m}$ thickness from four brain tissues with cyst and from three brain tissues without cyst were deparaffinized and rehydrated. Afterward, the endogenous peroxidase was blocked. Unmasking of the target molecules was performed using citrate buffer $(\mathrm{pH} 6$, Tween $0.05 \%$ ). Slides were incubated for 60 minutes in blocking solution containing $0.05 \%$ Triton X-100 in PBS supplemented with $10 \%$ horse serum and $5 \%$ bovine serum albumin. The sections were then incubated overnight with 1:200 goat anti-rat IgG antibodies that had been peroxidase labeled (KPL, Gaithersburg, MD) and diluted in 5\% goat normal sera in PBS-Triton X-100 0.05\%. The antigenantibody reaction was detected with chromogenic substrate diaminobenzidine (Dako, Carpinteria, CA), which exhibited a brown color in the presence of a positive reaction. This was then counterstained with hematoxylin. After dehydration in a series of graded ethanol dilutions $(70 \%, 90 \%$, and $100 \%$ ), the sections were then cleared with xylol and mounted using Entellan New mounting medium. Images were obtained using Leica microscopy.

\section{Statistical Analysis}

Fisher's exact test was used to evaluate the difference in the proportions of infected rats with anatomical point of infection (types 1 and 2), dose group, and age group. Inflammatory cell counts, collagen layer thickening, and angiogenesis were expressed as means $\pm \mathrm{SEM}$; the different degrees of spongy changes and perivascular infiltrates were presented in proportions. The Mann-Whitney $U$-test was used to compare the reactivity to VEGF (measured intensity in pixels) between brain tissue with and without cyst. The analysis was performed using Stata statistical software version 10 (StataCorp LP, College Station, TX), and $P<0.05$ was considered significant.

\section{Results}

\section{Brain Anatomical Point of Inoculation and Infection Dose}

In the first study, 12 rats were infected intracranially by the extraparenchymal route (type 1). No rats developed cysts when inoculated with 10 activated oncospheres, whereas two of three rats from each group that were inoculated with 20,30 , and 40 activated oncospheres developed one to three cysts each. The parasites were located in both extraparenchymal (submeningeal or ventricle) and intraparenchymal brain tissue (Figure 1).

In the second study, 84 rats were inoculated with increasing doses of activated oncospheres $(30,60,90,180,360$, or 720$)$ either extraparenchymally or intraparenchymally. After 4 months of infection, the cysts were found in the parenchymal and extraparenchymal (submeningeal or ventricle) brain tissue (Figure 1). No cysts were found in the control groups, and they were not included for statistical analysis. The proportion of infected rats varied from $16 \%$ to $100 \%$ in the type 2 inoculation (intraparenchymal) and from $50 \%$ to $83 \%$ in the type 1 
Table 1 Number of Rats Intracranially Infected with Different Activated Taenia solium Oncosphere Doses Using Extraparenchymal (Type 1) and Intraparenchymal (Type 2) Routes

\begin{tabular}{|c|c|c|c|c|c|c|c|}
\hline \multirow[b]{2}{*}{$\begin{array}{l}\text { No. of } \\
\text { oncospheres }\end{array}$} & \multicolumn{3}{|c|}{ Extraparenchymal route* } & \multicolumn{3}{|c|}{ Intraparenchymal route $^{\dagger}$} & \multirow[b]{2}{*}{$P^{\ddagger}$} \\
\hline & $\begin{array}{l}\text { No. }(\%) \text { of } \\
\text { rats with cysts/ } \\
\text { without cyst }\end{array}$ & $\begin{array}{l}\text { No. }(\%) \text { of } \\
\text { ELISA-positive rats }\end{array}$ & $\begin{array}{l}\text { No. }(\%) \text { of } \\
\text { WB-positive rats }\end{array}$ & $\begin{array}{l}\text { No. }(\%) \text { of } \\
\text { rats with cysts/ } \\
\text { without cyst }\end{array}$ & $\begin{array}{l}\text { No. }(\%) \text { of } \\
\text { ELISA-positive rats }\end{array}$ & $\begin{array}{l}\text { No. }(\%) \text { of } \\
\text { WB-positive rats }\end{array}$ & \\
\hline 0 & $0 / 6(0)$ & 0 & 0 & $0 / 6(0)$ & 0 & 0 & \\
\hline 30 & $3 / 3(50)$ & $1(33)$ & $1(33)$ & $6 / 0(100)$ & $5(83)$ & $3(50)$ & 0.05 \\
\hline 60 & $3 / 3(50)$ & $1(33)$ & $3(100)$ & $5 / 1(83)$ & $5(100)$ & $3(60)$ & 0.23 \\
\hline 360 & $3 / 3(50)$ & $3(100)$ & $2(67)$ & $5 / 1(83)$ & $3(60)$ & $4(80)$ & 0.23 \\
\hline 720 & $5 / 1(83)$ & $5(100)$ & $4(80)$ & $5 / 1(83)$ & $4(80)$ & $4(80)$ & 1.0 \\
\hline Total & $22 / 14(61)$ & $15 / 22(68)$ & $14 / 22(63)$ & $27 / 9(75)$ & $20 / 27(74)$ & $18 / 27(66.7)$ & 0.21 \\
\hline
\end{tabular}

*Type 1 inoculation at 2-mm depth.

'Type 2 inoculation at 4-mm depth.

${ }_{\ddagger} p$ value of the two-sample test of proportions of rats that develop cysticerci in the brain between intraparenchymal and extraparenchymal route of infection.

${ }^{\S}$ One rat was ELISA positive and WB negative.

"One rat was WB positive and ELISA negative.

ELISA, enzyme-linked immunosorbent assay; WB, Western blot.

route (extraparenchymal). There was no statistically significant difference in the proportion of rats that developed cysts among the number of oncospheres (dose groups) by the intraparenchymal or extraparenchymal routes of inoculation (Table 1). Similarly, the overall proportion of infected rats was not significantly different between the intraparenchymal inoculation (27 of $36,75 \%)$ and the extraparenchymal inoculation (22 of $36,61 \%$ ) (Table 1).

In addition, no significant difference was found in the number of brain cysts between dose groups for the intraparenchymal and extraparenchymal injection routes. In both groups (type 1 and type 2), approximately $80 \%$ of the infected rats, irrespective of the oncosphere dose, had only one cyst; the rest of the animals had two or more cysts (Table 2).

All the rat brain cysts found at necropsy were viable cysts with vesicular appearance, and no calcified cysts were detected macroscopically in any of the inoculated rats. The cysts were oval, were filled with translucent fluid, and contained a single white invaginated scolex, except for $8(80 \%)$ of the 10 cysts located in the ventricle that did not develop the scolex. There was no difference in the cyst morphologic characteristics between the intraparenchymal and the extraparenchymal route of inoculation, and all the cysts had similar macroscopic and microscopic (hematoxylin and eosin stain) (Figure 1) characteristics, with an outer cuticle layer and an inner layers. No cysts were observed in the rat skeletal muscle.

Circulating parasite antigen and antibodies were detected in the plasma of inoculated rats by ELISA and WB, respectively (Table 1). For the group inoculated intraparenchymally, 20 (74\%) of 27 rats that developed brain cysts tested positive on ELISA, and 18 (67\%) of 27 tested positive on WB. In the extraparenchymal group, 15 (68\%) of 22 rats with brain cysts tested positive on ELISA, and 14 (63\%) of 22 also tested positive on WB. One of the 14 necropsy-negative rats inoculated extraparenchymally tested positive on WB; similarly, in the intraparenchymal group, 1 of the 9 necropsy-negative rats tested positive on both ELISA and WB, and another one tested positive only on WB. The 12 serum samples from control animals tested negative on WB. The three CSF samples from infected rats tested positive to circulating parasite antigen, and the two controls tested negative.

\section{Rat Age at Infection}

We decided to challenge the rats extraparenchymally. In general, younger rats were found to have higher rates of infection than older rats. The infection rate decreased as the age of the rats increased; for instance, 10-day-old rats had an infection rate of $83 \%$ (15/18), whereas 18-day-old animals had an infection rate of $66 \%$ (6/9), and 26-day-old rats had an infection rate of only $25 \%(2 / 8)$. The difference in the

Table 2 Number of Cisticerci Developed in the Rat Brain Using Intraparenchymal or Extraparenchymal Route of Infection

\begin{tabular}{|c|c|c|}
\hline & $\begin{array}{l}\text { Intraparenchymal } \\
\text { route of infection }\end{array}$ & $\begin{array}{l}\text { Extraparenchymal } \\
\text { route of infection }\end{array}$ \\
\hline $\begin{array}{l}\text { No. of } \\
\text { cysts in } \\
\text { the rat brain* }\end{array}$ & $\begin{array}{l}\text { No. of rats/total } \\
\text { No. of infected } \\
\text { rats }(\%)\end{array}$ & $\begin{array}{l}\text { No. of rats/total } \\
\text { No. of infected } \\
\text { rats }(\%)\end{array}$ \\
\hline 1 & $22 / 27(81.5)$ & $17 / 22(78.3)$ \\
\hline 2 & $3 / 27(11.1)$ & $3 / 22(13)$ \\
\hline 3 & $1 / 27(3.7)$ & $1 / 22(4.4)$ \\
\hline 4 & $1 / 27(3.7)$ & $1 / 22(4.4)$ \\
\hline
\end{tabular}

*Rats that had two or more cysts; the cysts were intraparenchymal, extraparenquimal (ventricle or submeningeal), or both. 
Table 3 Cysticercosis Infection Rate in Rats at Different Ages Extraparenchymally Inoculated with Activated Taenia solium Oncospheres

\begin{tabular}{llrr}
\hline Age of rats (days) & No. of rats with/without cysts $(\%)$ & \multicolumn{1}{l}{ No. of cysticerci detected, range (median) } \\
\hline 10 & $15 / 3(83)$ & $1-11(3)$ & $P^{*}$ \\
18 & $6 / 3(67)$ & $1-7(1)$ & 0.35 \\
26 & $2 / 6(25)$ & $1(1)$ & $<0.01$ \\
\hline
\end{tabular}

${ }^{*} P$ value of the difference in proportion of infected rats using Fisher's exact test. It was different between rats of 26 days and 18 or 10 days of age.

Ref, reference group used to calculate the differences between groups.

proportion of infected rats was statistically different between the youngest and the oldest group $(P<0.01)$ (Table 3$)$.

Interestingly, 2 of the 23 infected rats (all ages) developed epilepsy at 5 months after infection. One rat (18-day-old group) had four viable cysts, two of them located in the ventricle and the other two in the parenchymal brain tissue (cortex and neocortex, hippocampus, and caudate putamen nucleus). The other rat (10-day-old group) had nine cysts; three of them (viable ones) were lodged submeningeally, and another six were in the parenchymal brain tissue (cortex and neocortex, hippocampus, caudate putamen nucleus, and cerebellum). One of the parenchymal cysts had a focal early degenerate cyst wall. The seizures were characterized as generalized tonic-clonic and were observed at least once a week during the last month before necropsy.

\section{Parasite Viability by in Vitro Cyst Evagination}

The ability of the organism to evaginate and develop into an adult tapeworm was evaluated from brain cysts. We selected a total of nine cysts located in the parenchymal brain tissue; six of them $(66.6 \%)$ successfully evaginated. Similarly, 11 (68\%) of 16 cysts located extraparenchymally evaginated as well. The scolex was absent in two nonevaginated cysts; however, they were PCR positive to T. solium.

\section{Histopathologic Study}

\section{Brain Location of Cysts}

The histopathologic analysis was performed on rats that developed only one brain cyst regardless of the infection route, the oncosphere dose, or the age of the rats. A total of 30 rats with unique cysts were located in the parenchyma (Figure 1A), primarily in the cortex and neocortex; the other anatomical locations were the hippocampus, caudate putamen nucleus, cerebellum, and corpus callosum. Another six animals had submeningeal cysts (Figure 1B). Finally, four rats had unique cysts inside the lateral ventricles (Figure 1C); one of them was attached to the ependymal cells, whereas the other three were floating in the ventricular space and did not have the scolex.

\section{Histologic Analysis of the Brain from Control Rats}

The brains from 12 control rats (extraparenchymal and intraparenchymal challenges) were histologically analyzed to compare with the infected groups. In these control brains, the distribution, morphologic features, and architecture of neurons appeared normal. Pyknotic cells, spongiform changes, mass effect, and perivascular infiltrate were not found. Masson's trichromic staining revealed no expression of collagen in the brain parenchyma, but a scant normal amount was found in the choroid plexus. There was no evidence of inflammation.

Histologic Analysis of Tissue Surrounding the $T$. solium Cyst The first layer observed immediately surrounding the cysts was a fibrotic tissue in all the submeningeal, ventricular, and parenchymal cysts (Figure 2A). It was thick and formed by dense collagen fibers (Table 4) and small vessels lined with amorphous endothelial cells.

Surrounding this layer, a variable degree of rarefaction and vacuolization of the neuropil in the brain parenchyma (spongy changes) (Figure 2B) was observed. These spongy changes had a width of 3 to $200 \mu \mathrm{m}$, and they were observed in all the 30 rat brains carrying unique parenchymal cysts (Figure 3). The severe condition was present only in parenchymal cysts and represented $10 \%$ from all these types of cysts. In addition, $33 \%$ of the submeningeal cysts had no spongy changes (Figure 3). No spongy changes were found in noninfected control rats.

The perivascular space (Virchow-Robin) expanded mainly by lymphoplasmacytic inflammatory infiltrative

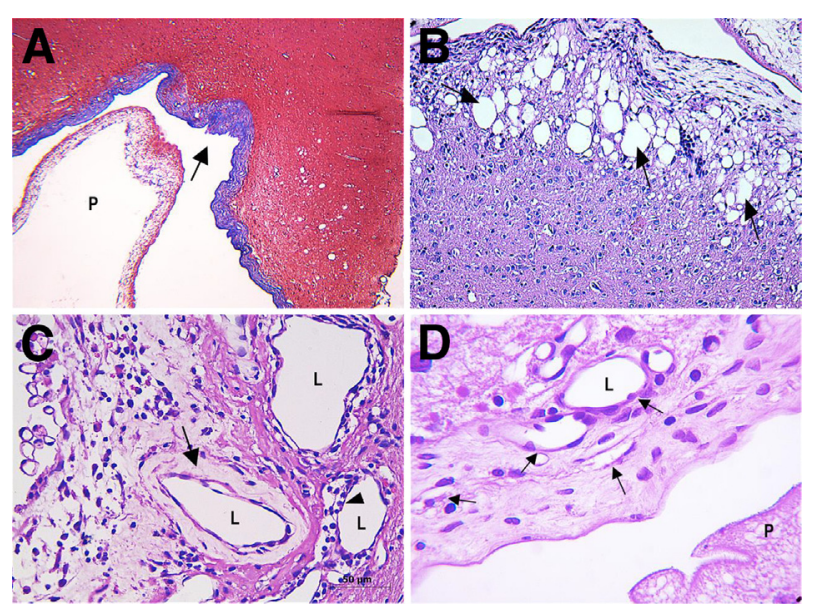

Figure 2 Rat brain tissue that developed cisticerci. A: Masson's trichromic stain to identify the collagen layer surrounded the cyst; the arrow indicates the collagen layer stain blue. B-D: Brain tissue hematoxylin and eosin stain. B: Spongy change; the arrows indicate the rarefaction and vacuolation of the neurophil in the brain tissue surrounding the cyst. C: The arrow indicates the thickened walls of small blood vessel, and the arrowhead indicates the perivascular infiltrate. $\mathbf{D}$ : The arrows indicate angiogenesis with small blood vessels spreading into fibrotic tissue. Scale bar $=$ $50 \mu \mathrm{m}$. Original magnification: $\times 4(B) ; \times 40(C$ and D). L, lumen; $P$, parasite. 
Table 4 Inflammatory Infiltrate Cells and Collagen Layer Thickness in NCC Rat Brain Tissue

\begin{tabular}{|c|c|c|c|c|c|}
\hline \multirow[b]{2}{*}{ Cyst anatomical location } & \multicolumn{4}{|c|}{ No. of each cell type* } & \multirow{2}{*}{$\begin{array}{l}\text { Thickness of collagen } \\
\text { layer }(\mu \mathrm{m})\end{array}$} \\
\hline & Heterophil $^{\dagger}$ & Lymphocytes & Plasmocytes & Macrophages & \\
\hline \multicolumn{6}{|l|}{ Control $^{\ddagger}$} \\
\hline Ventricle & $16.5 \pm 14.3$ & $24.5 \pm 15$ & $61 \pm 34$ & $47.8 \pm 21.3$ & $42.0 \pm 13^{\S}$ \\
\hline Submeningeal & $13.2 \pm 4.5$ & $13.7 \pm 7.6$ & $18.7 \pm 12.9$ & $19.0 \pm 10$ & $29.0 \pm 16^{\mathbb{\pi}}$ \\
\hline
\end{tabular}

*Rat brain tissues from 30 parenchymal cysts, 4 ventricle cysts, 6 submeningeal cysts, and 12 controls. Data presented as means \pm SEM.

${ }^{\dagger}$ Heterophil cells are neutrophil or eosinophil cells.

${ }^{\ddagger}$ No cells observed in the control group.

${ }^{\S} 0$ ne ventricular cyst had thicker collagen layer $(66 \mu \mathrm{m})$, and the cyst was attached to the ependymal layer.

ISubmeningeal cysts revealed meningeal fibrosis characterized by thickening and fusion of the pia and arachnoid mater.

cells. Perivascular inflammatory cuffs in brain vasculature were observed in areas surrounding the cyst (Figure 2C). The higher proportion of severe perivascular infiltrate was detected in one of the four ventricular cysts, where the cyst was attached to ependymal layer, whereas most of the parenchymal cysts had mild infiltrate (Figure 3).

Pyknotic cells were observed in brain tissue surrounding the ependymal layer in all the ventricle cysts. Similar changes were also observed in 28 (93\%) of the 30 parenchymal cysts and in five (67\%) of the six submeningeal cysts. Mass effect was observed in all the infected brains, causing compression and deviation of the median line between hemispheres. In ventricular cysts, this mass effect caused dilation of the ventricle lumen, leading to an internal hydrocephalus.

\section{Inflammatory Infiltrate Cells}

Infected rats had an increased number of heterophiles (eosinophils and neutrophils), macrophages (macrophages and activated microglia), plasmocytes, and lymphocytes compared with control rats. The infiltration of macrophages and plasmocytes was the most marked, followed by lymphocytes and heterophils (Table 4). In general, using Luna stain, we found that the mean \pm SEM percentage of eosinophiles from heterophil cells was $13 \% \pm 7 \%$.

\section{Angiogenesis}

Angiogenesis was observed with small blood vessels spreading into the fibrotic tissue and in the interface between fibrotic tissue and brain parenchymal tissue surrounding the parenchymal cyst in $100 \%$ of infected rats (Figure 2D). The mean \pm SD number of angiogenesis blood vessels surrounding the parenchymal cyst was $57 \pm 9$, and $27(6 \%)$ of 455 of them had thickened walls (Figure 2C); they stained moderately positive with Masson's trichromic stain.

\section{Immunohistochemical Analysis}

We found that reactivity to antibody against VEGF was higher in the brain tissue surrounded the cyst than in the brain tissue from control group $(P<0.0001)$ (Figure $4, \mathrm{~A}-\mathrm{C}$ ). Regarding the integrity of BBB, two of the four rat brains with cysts had a high reactivity to anti-rat IgG (Figure 4D), one tissue had weak reactivity and the other one was negative. In contrast, the three brain tissues from control group (no brain cysts) were negative.

\section{Discussion}

This study describes a novel neurocysticercosis model using rats infected intracranially with activated $T$. solium oncospheres. The cysts, or cysticerci, which developed exhibited characteristics identical to the ones observed in the natural hosts (humans and pigs). The cysts were located in the parenchymal or extraparenchymal (ventricles or meninges) brain tissue, as in natural hosts, and had similar morphologic characteristics (such as the scolex and vesicular cyst wall). Most infected rats (necropsy positive) developed specific antibodies against $T$. solium cysticerci (WB positive) and T. solium circulating antigens (ELISA positive), as do most natural hosts. ${ }^{25,26}$ There were a few rats that were WB and/ or ELISA positive but necropsy negative. It is likely that the cysts were located in other parts of the body, such as the spinal cord or muscles, but we were not able to find them.

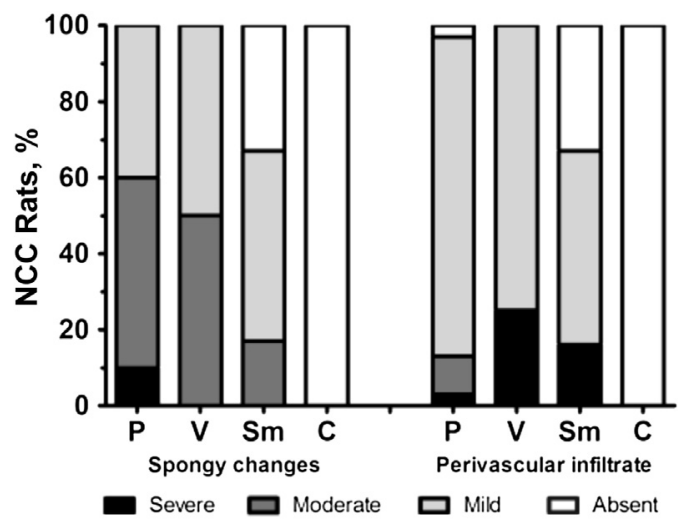

Figure 3 Proportion of neurocysticercosis (NCC) rats with spongy changes and perivascular infiltrate in the brain tissue surrounded the cyst. Spongy changes were classified as absent (no spongy changes observed), mild ( $<20 \%$ of the brain tissue was affected), moderate $(21 \%$ to $50 \%$ of the brain tissue affected), and severe $(>50 \%$ of the brain tissue showed alterations). Perivascular infiltrate was classified as absent (no infiltrates), mild ( $<20 \%$ of perivascular infiltration), moderate $(21 \%$ to $50 \%$ of perivascular infiltrates), and severe ( $>50 \%$ of these changes observed). C, control brain tissue; P, parenchymal cyst; Sm, submeningeal cyst; $\mathrm{V}$, ventricle cyst. 


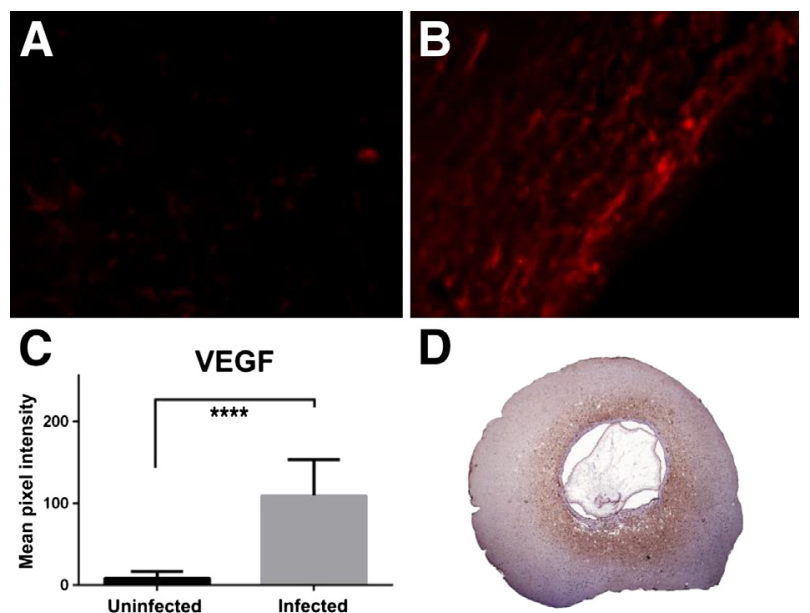

Figure 4 Immunohistochemistry of rat brain tissue. A and B: vascular endothelial growth factor (VEGF) expression using Alexa fluor 594 (red). Brain tissue without cyst $(\mathbf{A})$ and brain tissue surrounded the cyst with high reactivity to anti-VEGF (B). C: Quantitative measurements of VEGF. D: IgG extravasation in rat brain tissue using anti-rat IgG peroxidase labeled. Brain tissue surrounding the cyst reveals reactivity to anti-rat IgG (brown). $* * * * P<0.0001$ between tissues surrounded the cyst and tissue without cyst. Original magnification: $\times 40(\mathbf{A}$ and $\mathbf{B}) ; \times 2.5$ (D)

Although rat infection rates were not dose dependent, the rat age is crucial to the success of the infection and parasite load. Younger rats $(<19$ days old $)$ had higher rates of infection and higher parasite loads than older rats; it is probably related to the maturity of the rat immune response. ${ }^{31}$ Younger rats do not have a mature immune response, which is possibly why they were more likely to develop NCC with higher parasite loads.

Activated oncospheres successfully invaded the parenchymal and extraparenchymal regions of the brain. Even when inoculated extraparenchymally, the activated oncosphere can migrate through host brain tissue and develop into a metacestode. Mature cysticerci, as in previous experimental infection in pigs, ${ }^{24}$ were present 3 months after the challenge, with identical histologic characteristics. Most cysticerci were located in the parenchymal areas of the brain, as in human neurocysticercosis. ${ }^{32,33}$ In addition, the cyst viability was similar to that of cysts in the pig. ${ }^{23}$ Thus, the rat model seems to be an accurate model for studying the disease.

The infected rats developed ventricular ependymitis, meningitis, internal hydrocephalus, and spongy changes of the neuropil, which have also been reported in human and murine NCC. ${ }^{10,17,34,35}$ The angiogenesis observed in the brain tissue of infected rats has also been reported in human and porcine NCC. ${ }^{12,13,36}$ The angiogenesis process probably plays a role in promoting and regulating other biological events, such as inflammation, fibroblast proliferation, extracellular matrix synthesis, and BBB disruption, which have all been reported in others studies. ${ }^{29,37,38}$ It is still unknown what factors might be regulating the increased expression of VEGF in NCC; we hypothesize that some excretory-secretory parasite proteins may be regulating this phenomenon. Future works must be necessary to determine whether the parasite is fulfilling a role in the increased expression of VEGF.

Our study reveals the $\operatorname{IgG}$ extravasation in the tissue surrounded the cyst, demonstrating BBB disruption as mentioned in other studies ${ }^{28,29}$; however, it is essential to evaluate the alteration of BBB in NCC and understand the different inflammatory pathways that are mediating this process. The thickening of the blood vessels walls found around the cysts in infected rats has also been reported in biopsies of human brain tissue with NCC. ${ }^{39}$ This thickening is probably secondary to inflammation, which may increase susceptibility to breakage and have some role in the disruption of the BBB; further studies are necessary to explore this concept. Alterations in brain tissue architecture close to the cysticercus implantation site is probably likely due to physical compression caused by the parasite, the action of cysticercus-secreted molecules, and the inflammatory response of the host.

The inflammatory infiltrate cells of the rat NCC model is similar to human NCC; it includes the infiltration of high numbers of plasma cells, macrophages, $\mathrm{T}$ lymphocytes, and a low number of eosinophils. ${ }^{13,40}$ In rats, $T$. solium cysts and the resulting inflammatory process might induce the expression of neutrophil attractant chemokines as has been described in humans. ${ }^{40,41}$ In contrast, naturally infected porcine NCC has abundant eosinophils in the early stage of infection ${ }^{13}$; therefore, the rat model might be a good model to study the inflammatory process in humans.

Because there is a natural progression of infection from the oncosphere to metacestode stage in this model, it is possible to study the host's innate, early-induced, adaptive immune response and pathologic changes, which lead to the clinical manifestations of neurocysticercosis.

This model also provides a number of benefits over other animal models. First, we were able to obtain CSF, which might be used to detect different biomarkers at varying times after infection, such as parasite antigens, and cytokines. Second, it allows us, at low cost, to explore treatments that can then be screened in the more expensive natural pig model. Third, experimental infection of rat brains is not difficult, and the success rate of infection is high. Fourth, the rat is an ideal model in which to study epilepsy, a major clinical syndrome in human neurocysticercosis. Epilepsy has previously been studied in rats in the context of other diseases using electrophysiology, ${ }^{42}$ and those techniques could be applied to our rat NCC model. However, different clinical signs in pigs have been reported with cysticercosis but without epilepsy. ${ }^{43}$ Fifth, reagents to detect a variety of biomarkers are readily available for use in rats. Finally, rats are easy to handle in the laboratory.

There are relatively few limitations to this model. The rat is not the natural host, and the route of infection is not through the natural fecal-oral route. Also, because of the size of the rat brains, there could be an exacerbation of mass effect and inflammation caused by the $T$. solium cyst. 
In summary, analysis of the pathophysiologic features and the kinetics of the immune response in NCC patients is not possible, and alternative models are needed for research.

Therefore, the rat NCC model is a viable animal model to investigate the host immune response and test different drugs and scheme of treatment. This study presents a viable animal model for the study of human NCC.

\section{References}

1. Ndimubanzi PC, Carabin H, Budke CM, Nguyen H, Qian YJ, Rainwater E, Dickey M, Reynolds S, Stoner JA: A systematic review of the frequency of neurocyticercosis with a focus on people with epilepsy. PLoS Negl Trop Dis 2010, 4:e870

2. Mahanty S, Garcia HH: Cysticercosis and neurocysticercosis as pathogens affecting the nervous system. Prog Neurobiol 2010, 91:172-184

3. Villaran MV, Montano SM, Gonzalvez G, Moyano LM, Chero JC, Rodriguez S, Gonzalez AE, Pan W, Tsang VC, Gilman RH, Garcia HH: Epilepsy and neurocysticercosis: an incidence study in a Peruvian rural population. Neuroepidemiology 2009, 33:25-31

4. Adalid-Peralta L, Fleury A, Garcia-Ibarra TM, Hernandez M, Parkhouse M, Crispin JC, Voltaire-Proano J, Cardenas G, Fragoso G, Sciutto E: Human neurocysticercosis: in vivo expansion of peripheral regulatory $\mathrm{T}$ cells and their recruitment in the central nervous system. J Parasitol 2012, 98:142-148

5. Garcia HH, Del Brutto OH: Taenia solium cysticercosis. Infect Dis Clin North Am 2000, 14:97-119. ix

6. Garcia HH, Gilman RH, Gonzalez AE, Verastegui M, Rodriguez S, Gavidia C, Tsang VC, Falcon N, Lescano AG, Moulton LH, Bernal T, Tovar M: Hyperendemic human and porcine Taenia solium infection in Peru. Am J Trop Med Hyg 2003, 68:268-275

7. White AC Jr, Robinson P, Kuhn R: Taenia solium cysticercosis: hostparasite interactions and the immune response. Chem Immunol 1997, 66:209-230

8. Garcia HH, Del Brutto OH: Neurocysticercosis: updated concepts about an old disease. Lancet Neurol 2005, 4:653-661

9. Garcia HH, Modi M: Helminthic parasites and seizures. Epilepsia 2008, 49(Suppl 6):25-32

10. Sinha S, Sharma BS: Neurocysticercosis: a review of current status and management. J Clin Neurosci 2009, 16:867-876

11. Garcia HH, Lescano AG, Lanchote VL, Pretell EJ, Gonzales I, Bustos JA, Takayanagui OM, Bonato PS, Horton J, Saavedra H, Gonzalez AE, Gilman RH: Pharmacokinetics of combined treatment with praziquantel and albendazole in neurocysticercosis. Br J Clin Pharmacol 2011, 72:77-84

12. Sikasunge CS, Johansen MV, Phiri IK, Willingham AL 3rd, Leifsson PS: The immune response in Taenia solium neurocysticercosis in pigs is associated with astrogliosis, axonal degeneration and altered blood-brain barrier permeability. Vet Parasitol 2009, $160: 242-250$

13. Alvarez JI, Londono DP, Alvarez AL, Trujillo J, Jaramillo MM, Restrepo BI: Granuloma formation and parasite disintegration in porcine cysticercosis: comparison with human neurocysticercosis. J Comp Pathol 2002, 127:186-193

14. Sikasunge CS, Phiri IK, Phiri AM, Dorny P, Siziya S, Willingham AL 3rd: Risk factors associated with porcine cysticercosis in selected districts of Eastern and Southern provinces of Zambia. Vet Parasitol 2007, 143:59-66

15. Matos-Silva H, Reciputti BP, Paula EC, Oliveira AL, Moura VB, Vinaud MC, Oliveira MA, Lino-Junior Rde S: Experimental encephalitis caused by Taenia crassiceps cysticerci in mice. Arq Neuropsiquiatr 2012, 70:287-292

16. Khalifa RM, Teale JM, Mohamadain HS: Studies on some metacestodes immunohistochemical response in mice as a model for human cysticercosis: II-THI type immune response in experimental Braintaenia crassiceps infected mice. J Egypt Soc Parasitol 2012, 42: $183-190$

17. Cardona AE, Restrepo BI, Jaramillo JM, Teale JM: Development of an animal model for neurocysticercosis: immune response in the central nervous system is characterized by a predominance of gamma delta $\mathrm{T}$ cells. J Immunol 1999, 162:995-1002

18. Mishra PK, Morris EG, Garcia JA, Cardona AE, Teale JM: Increased accumulation of regulatory granulocytic myeloid cells in mannose receptor $\mathrm{C}$ type 1-deficient mice correlates with protection in a mouse model of neurocysticercosis. Infect Immun 2013, 81:1052-1063

19. Jeri C, Gilman RH, Lescano AG, Mayta H, Ramirez ME, Gonzalez AE, Nazerali R, Garcia HH: Species identification after treatment for human taeniasis. Lancet 2004, 363:949-950

20. Mayta H, Talley A, Gilman RH, Jimenez J, Verastegui M, Ruiz M, Garcia HH, Gonzalez AE: Differentiating Taenia solium and Taenia saginata infections by simple hematoxylin-eosin staining and PCRrestriction enzyme analysis. J Clin Microbiol 2000, 38:133-137

21. Verastegui M, Gilman RH, Arana Y, Barber D, Velasquez J, Farfan M, Chile N, Kosek JC, Kosek M, Garcia HH, Gonzalez A: Taenia solium oncosphere adhesion to intestinal epithelial and Chinese hamster ovary cells in vitro. Infect Immun 2007, 75:5158-5166

22. Nirogi R, Kandikere V, Mudigonda K, Bhyrapuneni G, Muddana N, Saralaya R, Benade V: A simple and rapid method to collect the cerebrospinal fluid of rats and its application for the assessment of drug penetration into the central nervous system. J Neurosci Methods 2009, 178:116-119

23. Gonzalez AE, Falcon N, Gavidia C, Garcia HH, Tsang VC, Bernal T, Romero M, Gilman RH: Treatment of porcine cysticercosis with oxfendazole: a dose-response trial. Vet Rec 1997, 141:420-422

24. Verastegui M, Gonzalez A, Gilman RH, Gavidia C, Falcon N, Bernal T, Garcia HH: Experimental infection model for Taenia solium cysticercosis in swine. Cysticercosis Working Group in Peru. Vet Parasitol 2000, 94:33-44

25. Tsang VC, Brand JA, Boyer AE: An enzyme-linked immunoelectrotransfer blot assay and glycoprotein antigens for diagnosing human cysticercosis (Taenia solium). J Infect Dis 1989, 159:50-59

26. Rodriguez S, Dorny P, Tsang VC, Pretell EJ, Brandt J, Lescano AG, Gonzalez AE, Gilman RH, Garcia HH: Detection of Taenia solium antigens and anti- antibodies in paired serum and cerebrospinal fluid samples from patients with intraparenchymal or extraparenchymal neurocysticercosis. J Infect Dis 2009, 199:1345-1352

27. Paxinos G, Watson C: The rat brain in Stereotaxic Coodinates. New York, Academic Press, 2007

28. Fukuda AM, Adami A, Pop V, Bellone JA, Coats JS, Hartman RE, Ashwal S, Obenaus A, Badaut J: Posttraumatic reduction of edema with aquaporin-4 RNA interference improves acute and chronic functional recovery. J Cereb Blood Flow Metab 2013, 33:1621-1632

29. Rigau V, Morin M, Rousset MC, de Bock F, Lebrun A, Coubes P, Picot MC, Baldy-Moulinier M, Bockaert J, Crespel A, LernerNatoli M: Angiogenesis is associated with blood-brain barrier permeability in temporal lobe epilepsy. Brain 2007, 130:1942-1956

30. Morin-Brureau M, Lebrun A, Rousset MC, Fagni L, Bockaert J, de Bock F, Lerner-Natoli M: Epileptiform activity induces vascular remodeling and zonula occludens 1 downregulation in organotypic hippocampal cultures: role of VEGF signaling pathways. J Neurosci 2011, 31:10677-10688

31. Katsuno R, Hasegawa T, Iwashina T, Sakai D, Mikawa Y, Mochida J: Age-related effects of cocultured rat nucleus pulposus cells and macrophages on nitric oxide production and cytokine imbalance. Spine (Phila Pa 1976) 2008, 33:845-849

32. Singh G, Rajshekhar V, Murthy JM, Prabhakar S, Modi M, Khandelwal N, Garcia HH: A diagnostic and therapeutic scheme for a solitary cysticercus granuloma. Neurology 2010, 75:2236-2245

33. Mitchell WG, Crawford TO: Intraparenchymal cerebral cysticercosis in children: diagnosis and treatment. Pediatrics 1988, 82:76-82 
34. Peeters D, Ceuterick C, De Jonghe P, Martin JJ: Cerebral cysticercosis. Light- and electron microscopy report on one case. Ann Soc Belg Med Trop 1980, 60:183-194

35. Srinivas HV, Rao TV, Deshpande DH: Cerebral cysticercosis: clinical and pathological observations with emphasis on the encephalitic type. Clin Neurol Neurosurg 1980, 82:187-197

36. Lino Junior Rde S, Ribeiro PM, Antonelli EJ, Faleiros AC, Terra SA, dos Reis MA, Teixeira Vde P: [Developmental characteristics of Cysticercus cellulosae in the human brain and heart] Portuguese. Rev Soc Bras Med Trop 2002, 35:617-622

37. Zhang ZG, Zhang L, Tsang W, Soltanian-Zadeh H, Morris D, Zhang R, Goussev A, Powers C, Yeich T, Chopp M: Correlation of VEGF and angiopoietin expression with disruption of blood-brain barrier and angiogenesis after focal cerebral ischemia. J Cereb Blood Flow Metab 2002, 22:379-392

38. Zhu Y, Lee C, Shen F, Du R, Young WL, Yang GY: Angiopoietin-2 facilitates vascular endothelial growth factor-induced angiogenesis in the mature mouse brain. Stroke 2005, 36:1533-1537
39. Lino-Junior Rde S, Faleiros AC, Vinaud MC, Oliveira FA, Guimaraes JV, Reis MA, Teixeira Vde P: Anatomopathological aspects of neurocysticercosis in autopsied patients. Arq Neuropsiquiatr 2007, 65:87-91

40. Restrepo BI, Alvarez JI, Castano JA, Arias LF, Restrepo M, Trujillo J, Colegial $\mathrm{CH}$, Teale JM: Brain granulomas in neurocysticercosis patients are associated with a Th1 and Th2 profile. Infect Immun 2001, 69:4554-4560

41. Garg RK, Malhotra HS: Solitary cysticercus granuloma. Expert Rev Anti Infect Ther 2012, 10:597-612

42. Navarro Mora G, Bramanti P, Osculati F, Chakir A, Nicolato E, Marzola P, Sbarbati A, Fabene PF: Does pilocarpine-induced epilepsy in adult rats require status epilepticus? PLoS One 2009, 4:e5759

43. Prasad KN, Chawla S, Prasad A, Tripathi M, Husain N, Gupta RK: Clinical signs for identification of neurocysticercosis in swine naturally infected with Taenia solium. Parasitol Int 2006, 55: $151-154$ 